\title{
VERB GO COMBINATIONS IN PERSPECTIVE ENGLISH LINGUISTICS AND CULTURE
}

\author{
Deden Novan Setiawan Nugraha \\ Faculty of Business and Management Widyatama University \\ deden.novan@widyatama.ac.id
}

\begin{abstract}
Language and culture are both integral parts of human life. Humans use language to express their feelings, and to communicate to each other. To support the processs of communication language must be able to represent ideas of speakers and be understood by the hearers. Linguistics is the study of languge. The part of linguistics that is concerned with the structure of language is divided into a number of subfields: syntax and semantics. Syntax is the system of rules and categories that underlies sentence formation in human language, meanwhile semantics is the study of meaning in human languge. Because language is such a central feature of being a human, linguistics has connections with many other disciplines in the humanities like culture. Based on Sapir-Whorf Hypothesis (Sapir, 1929); (a). Language influences thought and culture; (b). Language and culture influence each other. This study aims to investigate syntactic categories combined to the verb go and to analyze the meaning of the verb go after being combined with the complements. The data itself is taken from the British National Corpus (BNC). The results of the article indicate that there is a very close relationship between language and culture. That is, culture has a direct effect on language. Language and culture are closely correlated. Based on the analysis, the verb go can be combined with nouns, verbs, adjectives, adverbs, verbs-ing and past participles. In the case of semantic analysis there are two categories of meaning of the verb go combinations: dynamic verb indicates moving away from something or causing something to move or to happen; and stative verb which shows the meaning of steady state with no internal changes or phases. Most of the verb go combinations have generates new meaning, that is idiomatic meaning.
\end{abstract}

\section{Keywords: Verb go Combinations, Syntactic, Semantics, Culture.}

\section{Introduction}

In order to understand the specific words, literary terms, and even sometimes the simple words in one language, we must be familiar with the culture of that nation. In English, to understand a sentence, we have to know the structure of the sentence since the meaning of a sentence is determined by the meaning of its components and the manner in which they are arranged in syntactic structure (O’Grady, 1993:224). One of the important elements of a sentence is a verb. It plays very important role in sharing the complete meaning to the sentence. In the case of the form, the English verb is divided into two major forms "regular and irregular". The verb go belongs to the irregular verb. The verb is called irregular if the verb form changes without adding -ed for the past tense (preterit) and past participle. The forms of the verb go are as follows: to go, go, goes, going, went, gone. The forms like to go, going and gone are called non finite verbs, while the form like go, goes, and went are called finite verbs. The English clause consists of a subject and a finite verb: e.g.

(1) $\frac{\text { The milk }}{\mathrm{S}} \frac{\text { went }}{\mathrm{V}} \frac{\text { sour. }}{\mathrm{C}}$

The verb go belongs to the intransitive verb. A verb is called transitive if it needs an object, and intransitive verbs do not need objects: e.g.

(2) $\underline{\text { He }}$ killed a bird. (transitive)

$$
\mathrm{S} \mathrm{V} \quad \mathrm{O}
$$

(3) The company went bankrupt last year (intransitive)

$\begin{array}{llll}\mathrm{S} & \mathrm{V} & \mathrm{O} & \mathrm{Adv}\end{array}$


The verb go function as the main verb in the clause or an auxiliary showing something will happen in the future, e. g.

(4) The students went to the zoo. (main verb)

(5) We are going to spend our holiday in Singapore. (auxiliary)

From the semantic point of view, the verb go has a variety of meanings:

(6) Dad went first. (dead)

(7) The old tires finally went. (damaged)

(8) That country must go. (destroyed)

\section{Formulation of the Study}

1. What kinds of syntactic categories are combined to the verb go in multi-word combinations?

2. What is the meaning of the verb go after being combined with the complements?

\section{Methodology}

\subsection{The Method and Technique of Data Collecting}

This study uses the descriptive method that is a method which uses the empirical facts in order to get factual result.

\subsection{The Method and Study Technique.}

The method used is descriptive with substitution technique.

\section{Theoretical Framework 4.1 Syntax}

O'Grady (1993:156) said that syntax is the system of rules and categories that underlies sentence formation in human language.

\subsection{Syntactic Units}

In English, syntactic units like sentence, clause, phrase, and word.

\subsection{Syntactic Functions}

There are some words serving some functions in clauses. The function is called syntactic functions. There are subject, predicate, object and complement.

\subsection{Syntactic Categories}

A fundamental fact about words in all human languages is that they can be grouped together into a relatively small number of classes, called Syntactic Categories such as noun, verb, adjective, and adverb, determiner, preposition, conjunction and auxiliary. O’Grady, 1993:182).

\subsection{Multi-word Verb}

The followings are the definition of Multiword verb according to some linguists: " $A$ verb followed by a morphologically invariable particle, which functions with the verb as a single grammatical unit and behaves to some extent either lexically or syntactically as a single verb". (Quirk, et al., 1985:1150)

The quotation above means that a verb can have certain particle which can't be change with others. For examples: refer to, you can't say refer not; consist of, you can't say consist on. And such verbs have to be considered as a single verb.

"Multi-word verbs are a large and important class of English verbs in which a single verb-word works in close association with one or more other words. Most often there is just one other word, a 'particle,' in addition to the verb-word. This particle can be either a preposition or an adverb. Sometimes, however, the associated word is not a particle but rather an adjective, a noun, or another verb". (Claridge, 2000:145)

Claridge said that they are many English verbs have to be considered as one verb although these verb are followed by any others syntactic categories.

A multi-word verb is to see that it all shares the characteristic of unity. To say that a multi-word verb is unified is to say that the two or more words make up a single thinga unit. And because they are units, these verbs should be taught and learned as units. The nature of the unity, however, varies sharply between the two most important types - phrasal verbs and prepositional verbs. The unity of the phrasal verbs is primarily semantic. For example, when the phrasal verb "take off" is used in a sentence 
like "Jill took off her sweater" the verbword "take" loses the meaning it has when used alone and now works together with the particle "off" to create a new meaning. In the case of most prepositional verbs, by contrast, the verb-word and the particle form a syntactic not a semantic unit: the particle/preposition is required in order for an object to be attached to the verb. For example, when the prepositional verb "approve of" is used in a sentence like "Sam did not approve of Jill's behavior" the verb-word "approve" has the same meaning as it does when it is used without a preposition in the sentence "Sam did not approve."

According to Biber et al (1999:403) there are three major kinds of multi-word combinations that comprise relatively idiomatic and function like single verbs:

\section{Phrasal Verbs}

Phrasal verb is one of the categorizations of multi-word verb which consists of a lexical verb plus particle. In phrasal verb, the particle is an adverb. Aarts and Aarts (1999:427) states that phrasal verb is the combination of a verb with an adverb as the particle and they set as a single meaningful unit of the verb, the particles function like modifiers and usually cannot be separated in structure meaning.

Phrasal verbs may appear in transitive and intransitive types. As mentioned before transitive means the verb requires an object and intransitive means the verb do not require object.

\section{(9) I don't like to get up.}

From the example above (9) get up is a phrasal verb in intransitive type because consist of a verb get followed by adverbial particle $u p$ then the verb do not require the object. The meaning of get up is rise from bed.

\section{Prepositional Verbs}

Biber et al. (1999:442) defines a prepositional verb consists of lexical verb followed by a preposition with which it is semantically and/or syntactically associated. Because a preposition always has an object, all prepositional verbs have direct objects. Here are some examples of prepositional verbs:

\section{(10) I believe in God.}

From the example above (10) believe in is a prepositional verb because it consists of a verb believe followed by a preposition in. The meaning of believe in is have faith in the existence of. Because prepositional verbs end with a preposition in, there is always a direct object; the direct object is God.

\section{Phrasal Prepositional Verbs}

Biber et al (1999:423) defines Phrasal prepositional verbs consist of a lexical verb combined with an adverbial particle plus a preposition. As with prepositional verbs, the complement of the preposition in this construction functions as the direct object of the phrasal prepositional verbs. Consider the example:

(11) He doesn't get on with his wife.

From the example above (11) get on with is a phrasal prepositional verb because it consists of a verb get combined with adverbial particle on plus a preposition with. The meaning of get on with is having a friendly relationship with. Because phrasal prepositional verbs end with a preposition with, there is always a direct object; the direct object is his wife.

\subsection{Semantics}

O'Grady, 1993:211) states that semantics is the study of meaning in human language.

\subsection{Semantics of the Main Verbs}

Quirk et al. (1985:178) highlights that main verbs semantically can be classified into two classes: stative and dynamic. Stative verbs like be, have, know and love allow the speaker to view a situation as a steady state, with no internal phases or changes, it does not occur with the progressive. Moreover the speaker does not overtly focus on the beginning or the end of the state. A dynamic verb is a verb that shows continued or progressive action on the part of the subject. This is the opposite of a stative verb.

\section{Discussion}

\subsection{Stative Verb}

The combination of verb go can be followed by syntactic categories. In this section, the writer analyzes data which are included in verb, adjective, adverb, verb-ing, past participle and groups them into semantic 
meanings. One of the meanings which can be conveyed by multi-word verb go combination is stative verb.

\subsubsection{Verb Go followed by Verb}

(9) Data [92]

Medicare is on track to go broke by 2017 and our long term budget problems are primarily health cost problems.

Analysis: Syntactically, the data above consist of two clauses. The constituents are described as follows: The first clause is Medicare is on track to go broke by 2017. The subject is Medicare, the verb is is, the adverb is on track, the verb is to go which followed by the verb broke in past tense form functioning as the complement of the verb go and the adverb of time is by 2017. The second clause is and our long term budget problems are primarily health cost problems. The conjunction is and, the subject is our long term budget problem, the verb is are, the complement is primarily health cost problems. The first clause contain the verb go which is followed by the verb broke in past tense form as the focus of this research. The verb go in this clause is an intransitive verb that does not need an object. Semantically, the combination of the verb go with the verb broke in past tense form set as a single meaningful unit of the verb go and usually cannot be separated in structure meaning or it cannot be interpreted word by word but it has to be understood as whole. This combination generates an idiomatic meaning, in this case the combination of go broke means having no money or bankrupt. Based on the data above go broke is stative verb because it shows of steady of a state with no internal changes phases. In general the verb $g o$ is dynamic verb because it can be put in the progressive form, but in this data it is impossible to put the verb go into progressive form because it is preceded by the grammatical sign to. Therefore it is not acceptable to say: * Medicare is on track to going broke by 2017 and our long term budget problems are primarily health cost problems. By means the context, the meaning of the verb go here can be substituted by become. So the clause can be rewritten: Medicare is on track to become broke by 2017 and our long term budget problems are primarily health cost problems.

\subsubsection{Verb Go followed by Adjective}

(10) Data [239]

If you start to get too many things in your head, you will go crazy.

Analysis: Syntactically, the data above consist of two clauses. The constituents are described as follows: The first clause is a conditional clause: If you start to get too many things in your head. The subject of this clause is you which is preceded by conjunction if and the verb is start to get, while too many things in your head function as the direct object to the verb. The second clause is you will go crazy. The subject of this clause is you, will is the auxiliary modal verb, go is the verb while the crazy is an adjective functioning as the complement of the verb go. The second clause contain the verb go which is followed by an adjective crazy as the focus of this research. The verb $g o$ in this clause is an intransitive verb that does not need an object. Semantically, the combination of the verb go with the adjective crazy set as a single meaningful unit of the verb go and usually cannot be separated in structure meaning or it cannot be interpreted word by word but it has to be understood as whole. This combination generates an idiomatic meaning, in this case the combination of go crazy means insane or unbalanced, especially as manifested in wild or aggressive behavior. Based on the data above go crazy is stative verb because it shows of steady of a state with no internal changes phases. In general the verb go is dynamic verb because it can be put in the progressive form but in this data it is impossible to put the verb go into progressive form because the preceding sentence is in the present tense. If the preceding clause is in the present tense the following clause has to be in the present future tense. Therefore it is not acceptable to say: * If you start to get too many things in your head, you will going crazy. By means the context, the meaning of the verb go here can be substituted by become. So the clause can be rewritten: If you start to get too many things in your head, you will become crazy.

\subsubsection{Verb Go followed by Adverb}

(11) Data [91] 
The people who dedicate their lives to studying illness, go deep into debt for the privilege of caring for patients and work long hours.

Analysis: Syntactically, the data above consists of two clauses. The constituents are described as follows:The first clause is the people who dedicate their lives to studying illness. The subject is the people, who is the relative pronoun, dedicate is the verb, their lives is an object and to studying illness is the complement. The second clause is go deep into debt for the privilege of caring for patients and work long hours. Go is the verb, deep into debt for the privilege of caring for patients and work long hours are the complement. The second clause contain the verb go which is followed by an adjective deep functioning as a complement as the focus of this research. The verb go is an intransitive verb that does not need an object. Semantically, the combination of the verb go with the adverb deep set as a single meaningful unit of the verb go and usually cannot be separated in structure meaning or it cannot be interpreted word by word but it has to be understood as whole. This combination generates an idiomatic meaning, in this case the combination of $g o$ deep means far down. Based on the data above go deep is stative verb because it shows of steady of a state with no internal changes phase. By means the context, the meaning of the verb go here can be substituted by move. So the clause can be rewritten: The people who dedicate their lives to studying illness, move deep into debt for the privilege of caring for patients and work long hours.

\subsubsection{Verb Go followed by Verb-ing.}

(12) Data [69]

My dad lets me go tanning quite a bit.

Analysis: Syntactically, the data above is the simple sentence or a clause. The constituents are described as follows: The subject is my dad, the verb is lets, the object is $m e$, the verb is $g o$ which is followed by a noun tanning functioning as a complement of the verb go in the form of gerund, quite a bit is the adverb of time. The clause contain the verb $g o$ which is followed by a noun tanning functioning as a complement of the verb $g o$ in the form of gerund as the focus of this research. The verb go is an intransitive verb that does not need an object. Semantically, the combination of the verb go with a noun tanning functioning as a complement of the verb go in the form of gerund set as a single meaningful unit of the verb $g o$ and usually cannot be separated in structure meaning or it cannot be interpreted word by word but it has to be understood as whole. This combination generates an idiomatic meaning. In this case the combination of go tanning means a golden brown shade of skin developed by paleskinned people after exposure to the sun. This combination shows stative verb because it shows the meaning of steady state with no internal changes or phases. In general the verb go is dynamic verb because it can be put in the progressive form, but in this data it is impossible to put the verb go into progressive form because it is preceded by the verb lets. Therefore it is not acceptable to say: *My dad lets me going tanning quite a bit .By means the context, the meaning of the verb go here can be substituted by become. So the clause can be rewritten:

My dad lets me become tanning quite a bit.

\subsubsection{Verb Go followed by Past Participle}

(13) Data [33]

First, that it was discovered, since many such incidents go unnoticed, "security expert says".

Analysis: Syntactically, the data above consist of three clauses. Consider:The first clause is First, that it was discovered. The subject is it, the verb is was, while discovered function as the complement the form of past participle, so this clause is passive. The second clause is an independent clause dependent to the first by means of subordinate conjunction since many. The second clause is since many such incidents go unnoticed. The third clause is Security expert says. The subject is Security expert, the verb is says. The second clause content the verb go which is followed by unnoticed functioning as a complement of the verb go in the form of past participle as the focus of this research which is an intransitive verb that does not needs an object. Semantically, the combination of the verb go with 
unnoticed functioning as a complement of the verb $g o$ in the form of past participle set as a single meaningful unit of the verb go and usually cannot be separated in structure meaning or it cannot be interpreted word by word but it has to be understood as whole. This combination generates an idiomatic meaning. In this case the combination of $g o$ unnoticed means no pay attention. This combination shows stative verb because it shows the meaning of steady state with no internal changes or phases. In general the verb go is dynamic verb because it can be put in the progressive form. *First, that it was discovered, since many such incidents are going unnoticed, "security expert says". Therefore by means context, the meanings of the verb go here can be replaced by the verb happen. So the clause can be rewritten: First, that it was discovered, since many such incidents happen unnoticed, "security expert says".

\subsection{Dynamic Verb}

In this section, the writer analyzes data which are included in noun, verb, adjective, adverb, verb-ing, and groups them into semantic meanings.

\subsubsection{Verb Go followed by Noun}

(14) Data [28]

If you turn on a pitch and get all of it, you can go places.

Analysis: Syntactically, the data above consist of three clauses. The constituents are described as follows:The first clause begins with conjunction if, the subject is you, the verb is turn on, the object is a pitch. The second clause is and get all of it. Preceded with conjunction and, the verb is get, the object is all of it. The third clause is you can go places. The subject is you, the auxiliary modal verb is can, the verb is go followed by the noun places functioning as the complement of the verb $g o$. The third clause contain the verb go which is followed by the proper noun places as the focus of this research. The verb $g o$ in this clause is an intransitive verb that does not need an object. Semantically, the combination of the verb go with the proper noun places set as a single meaningful unit of the verb $g o$ and usually cannot be separated in structure meaning or it cannot be interpreted word by word but it has to be understood as whole. This combination shows dynamic verb because indicates moving away from something or causing something to move or to happen. Go places means to go somewhere or to move in particular way or to do something as you are moving. In general the verb go is dynamic verb because it can be put in the progressive form, but in this data it is impossible to put the verb go into progressive form because it is preceded by the auxiliary modal verb can. Therefore it is not acceptable to say: * If you turn on a pitch and get all of it, you can going places. By means the context, the meaning of the verb go here can be substituted by travel. So the clause can be rewritten: If you turn on a pitch and get all of it, you can travel places.

\subsubsection{Verb Go followed by Verb}

(15) Data [232]

Sauce splashes on my shirt. He tells me to go change; he'll take care of everything.

Analysis: Syntactically, the data above consist of three clauses. The constituents are described as follows:The first clause is Sauce splashes on my shirt. The subject is Sauce, the verb is splashes, the adverb is on my shirt. The second clause is He tells me to go change. The subject is $H e$, the verb is tells, the object is me, the verb is to go which followed by the verb change functioning as the complement of the verb go. The third clause is he'll take care of everything. The subject is $\mathrm{He}$, the auxiliary modal verb is will, the verb is take care, and the adverb is of everything. The second clause contain the verb go which is followed by the verb change as the focus of this research. The verb go in this clause is an intransitive verb that does not need an object. Semantically, the combination of the verb go with the verb change set as a single meaningful unit of the verb go and usually cannot be separated in structure meaning or it cannot be interpreted word by word but it has to be understood as whole. This combination generates an idiomatic meaning, in this case the combination of $g o$ change means replace the shirt become new. Based on the data above go change is dynamic verb because it causing moving away from something or causing something to move or to happen. 
In general the verb go is dynamic verb because it can be put in the progressive form, but in this data it is impossible to put the verb go into progressive form because it is preceded by the grammatical sign to. Therefore it is not acceptable to say: * Sauce splashes on my shirt. He tells me to going change; he'll take care of everything.

\subsubsection{Verb Go followed byAdjective}

(16) Data [289]

Sonny against the wall, cupping her ass in his hands. "I'll go slow," he promised

Analysis: Syntactically, the data above consist of two adverb phrases and two clauses. The constituents are described as follows: The first phrase is the adverb phrase of place: against the wall. The second phrase is the adverb phrase of manner: cupping her ass in his hands. The first clause is I'll go slow. I is the subject, will is the auxiliary modal verb, go is the verb followed with an adjective slow functioning as the complement of the verb go. The second clause is he promised. The subject is $h e$, the verb is promised. The first clause contain the verb go which is followed by the adjectives slow as the focus of this research. The verb go in this clause is an intransitive verb that does not need an object. Semantically, the combination of the verb go with the adjective slow set as a single meaningful unit of the verb go and usually cannot be separated in structure meaning or it cannot be interpreted word by word but it has to be understood as whole. This combination generates an idiomatic meaning, in this case the combination of $g o$ slow means moving at low speed. Based on the data above go slow is dynamic verb because it causing moving away from something or causing something to move or to happen. In general the verb go is dynamic verb because it can be put in the progressive form, but in this data it is impossible to put the verb go into progressive form because it is preceded by the auxiliary modal verb will. Therefore it is not acceptable to say:

* Sonny against the wall, cupping her ass in his hands. "I'll going slow," he promised. By means the context, the meaning of the verb go here can be substituted by move. So the clause can be rewritten: Sonny against the wall, cupping her ass in his hands. "I'll move slow," he promised

\subsubsection{Verb Go followed by Adverb}

(17) Data [149]

I just heard we were voting four times. Is it possible to go back to Washington?

Analysis: Syntactically, the data above consist of three clauses. The constituents are described as follows: The first clause is $I$ just heard. The subject is $I$, the adverb is just, the verb is heard. The second clause is we were voting four times. The subject is we, the verb is were voting, the adverb is four times. The third clause is in question form. Is it possible to go back to Washington? The verb is is, the subject is it, the complement is possible, the verb is to go followed with the adverb back to Washington functioning as the complement of the verb go. The third clause contain the verb go which is followed by the adverb back as the focus of this research. The verb go in this clause is an intransitive verb that does not need an object. Semantically, the combination of the verb go with the adverb back set as a single meaningful unit of the verb go and usually cannot be separated in structure meaning or it cannot be interpreted word by word but it has to be understood as whole. This combination generates an idiomatic meaning, in this case the combination of $g o$ back means in return. Based on the data above go back is dynamic verb because it causing moving away from something or causing something to move or to happen. In general the verb go is dynamic verb because it can be put in the progressive form. But in this data it is impossible to put the verb go into progressive form because it is preceded by the grammatical sign to. Therefore it is not acceptable to say: *. I just heard we were voting four times. Is it possible to going back to Washington? By means the context, the meaning of the verb go here can be substituted by move. So the clause can be rewritten: I just heard we were voting four times. Is it possible to move back to Washington?

\section{Conclusion}

From the mentioned points and discussion, it can be concluded that there is a very close relationship between language and culture in general, and a specific language and its culture in particular. The data shows that the 
kinds of syntactic categories can be combined to the verb go in multi-word combinations are nouns, verbs, adjectives, adverbs, verbs-ing and past participles. In the case of semantic analysis, most of the data shows that the verb go after being combined with the complements set as a single meaningful unit of the verb $g o$ and usually cannot be separated in structure meaning or it cannot be interpreted word by word but it has to be understood as whole and generates an idiomatic meaning. There are two categories of meaning the verb go after being combined with the complements. They are dynamic verb indicates moving away from something or causing something to move or to happen; and stative verb which shows the meaning of steady state with no internal changes or phases.

\section{References}

[1] Aarts, Flor and Jan Aarts. 1988. English Syntactic Structures, Function and Categories in Sentence Analysis. London: Prentice Hall and Martinus Nijhoff.

[2] Biber, Douglas et al.1999. Longman Grammar of Spoken and Written English. England: Pearson Education Limited.

[3] Claridge, Claudia.2000. Multi-word verbs in Early Modern English: A corpus-based study. Language and Computers: Studies in Practical Linguistics, 32. Amsterdam and Atlanta, GA.

[4] O'Grady, William et al. 1993. Contemporary Linguistics: An Introduction. Canada: Corp. Clark Pitman, Ltd.

[5] Quirk, Randolph et al. 1985. A Comprehensive Grammar of the English Language. England: Longman Group UK Limited.

[6] Sapir, E. (1929). The status of linguistics as a science. Language, 5, 207-214. 